\title{
A Twist-Bend Stage in the Relaxation of Sheared Chiral Nematic Suspensions of Cellulose Nanocrystals
}

Derek G. Gray ${ }^{1 *}$ and Xiaoyue $\mathrm{Mu}^{1}$

${ }^{1}$ Department of Chemistry, Pulp and Paper Building, 3420 University Street, Montreal, QC, Canada H3A 2A7

* Author to whom correspondence should be addressed; E-Mail: derek.gray@mcgill.ca; Tel.: +1-514-398-6182.

\section{Colours expected for twist-bend nematic structure when viewed with a polarized light microscope between crossed polars with $530 \mathrm{~nm}$ red plate}

For a conical helicoidal structure with axis along the $\mathrm{z}$ direction of a right-handed Cartesian coordinate frame, the director of a twist-bend structure, $\overline{\boldsymbol{n}}$, is defined by

$\overline{\boldsymbol{n}}=(\bar{x}, \overline{\boldsymbol{y}}, \overline{\boldsymbol{z}})=\left(\sin \theta_{0} \cos \varphi, \quad \sin \theta_{\circ} \sin \varphi, \cos \theta_{\circ}\right)$

where $\theta_{0}$ is the constant oblique angle (the tilt angle) of the director with the helix axis, $\mathrm{z}$, and $\varphi$ is the azimuthal angle of the oblique helicoid. $\varphi=2 \pi(z / P)=t z$ where $t=2 \pi / P$ and $P$ is the pitch of the conical helicoid ${ }^{1}$. Note that equation 1 also describes the nematic phase $\mathrm{N}$ when $\theta_{0}=0$, and the chiral nematic phase $\mathrm{N}^{*}$ when $\theta_{0}=\pi / 2$.

To examine how this might affect the texture of a twist-bend structure observed by polarized light microscopy, equation 1 may be used to calculate the director orientation for some arbitrary values of the tilt angle, $\theta_{0}$.

Consider one pitch length along the $z$ direction, corresponding to an increase in the azimuthal angle $\varphi$ from 0 to $2 \pi$ radians, and divide it arbitrarily into 16 steps. Starting at the origin of each step, the $x, y$ and $z$ co-ordinates of the end of the unit vector were calculated for tilt angles $0^{\circ}$, $15^{\circ}, 45^{\circ}, 75^{\circ}$ and $90^{\circ}$. A section of the conical helicoid viewed in the $x z$ plane is sketched in Figure S1. 


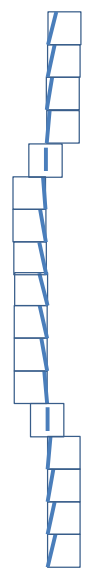

(b)

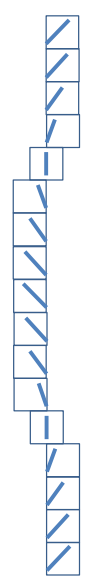

(c)

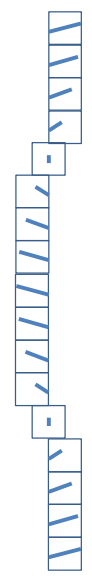

(d)

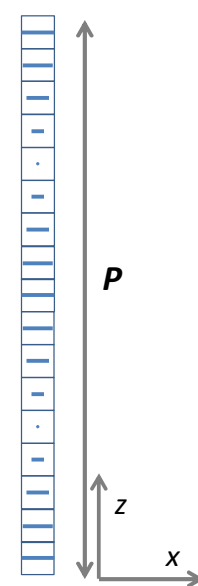

(e)
Figure S1. Sketch of projection on $x z$ plane of twistbend director orientation for one pitch length of helix, with fixed values for the tilt angle $\theta_{0}$ of (a) $\theta_{0}=$ $0^{\circ}$ (nematic); (b) $\theta_{\mathrm{o}}=15^{\circ}$; (c) $\theta_{\mathrm{o}}=45^{\circ}$; (d) $\theta_{\mathrm{o}}=75^{\circ}$; (e) $\theta_{\mathrm{o}}=90^{\circ}$ (chiral nematic). The pitch $P$ was arbitrarily divided into 16 small steps, and the orientation and length of the projection for each step was calculated from eq. 1.

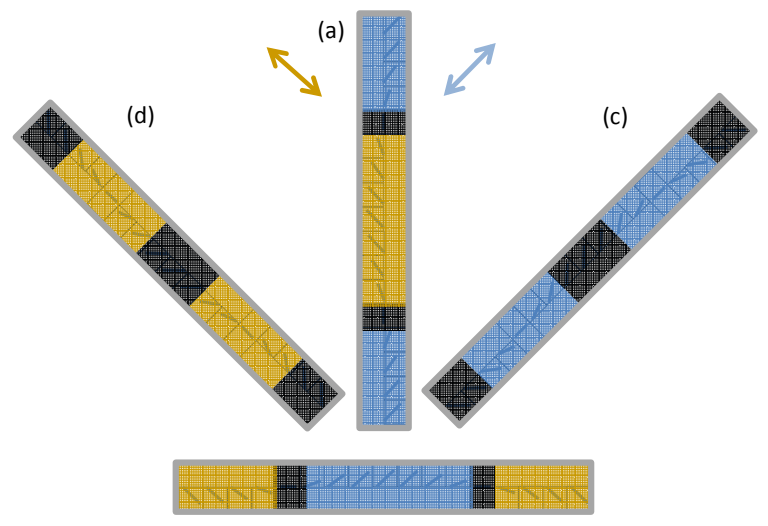

(b)

Figure.S2. Illustration of colours expected for projection on $x z$ plane of twist-bend director with tilt angle $\theta_{0}=45^{\circ}$. The yellow and blue arrows show the colours expected for positive birefringence in the indicated directions. The colours depend on the local twist-bend orientation, which in turn depends on the orientation of the twist-bend axis: for (a) and (b), the twist-bend axes are aligned parallel to the microscope polarizers, and for (c) and (d) the axes are aligned at $45^{\circ}$, as shown. Black indicates orientation of the parallel to the microscope polarizers.

The orientation of the unit vectors shown in Figure S1 may be converted to the colours generated by viewing with cross polars and $530 \mathrm{~nm}$ red plate, as shown for a tilt angle of $45^{\circ}$ in Figure S2. The observed pattern of blue, yellow and dark regions depends on the orientation of the twistbend helix axis relative to the orientation of crossed polars and wave plate. The patterns for four different orientations of the helix axis are shown. It is assumed that the helix axes are in the plane of the sample. When the conical helicoid axis is aligned with the crossed polars (a) and (b), the sample is expected to show yellow and blue bands of equal width, separated by dark lines. When the axis is at \pm 45 (c), (d), blue or yellow regions are separated by dark regions.

The patterns generated by a range of tilt angles and helix axis directions are shown in Figure S3. For tilt angle $\theta_{\mathrm{o}}=0^{\circ}$, the colour pattern is that for a uniform orientation along the nematic axis. For tilt angle $\theta_{0}=90^{\circ}$, the colours reverse, and black lines appear at separation of $P / 2$. This corresponds to the chiral nematic fingerprint pattern. The colour patterns for intermediate values of the tilt angle are more complex, with alternating bands of colour that change with helix axis orientation. One important observation is that for the twist-bend structures $0^{\circ}<\theta_{\mathrm{o}}<90^{\circ}$ the widths of the coloured bands are not in general constant, but fluctuate widely with helix axis 
orientation. This contrasts with the chiral nematic case, where the spacing of the fingerprint lines is constant for helix axes in the plane of the sample. This is taken as evidence compatible with a twist-bend intermediate structure, in analogy with the asymmetric or incomplete "Bouligand arcs" observed for bent molecule twist-bend phases by freeze-fracture transmission electron microscopy ${ }^{1}$.
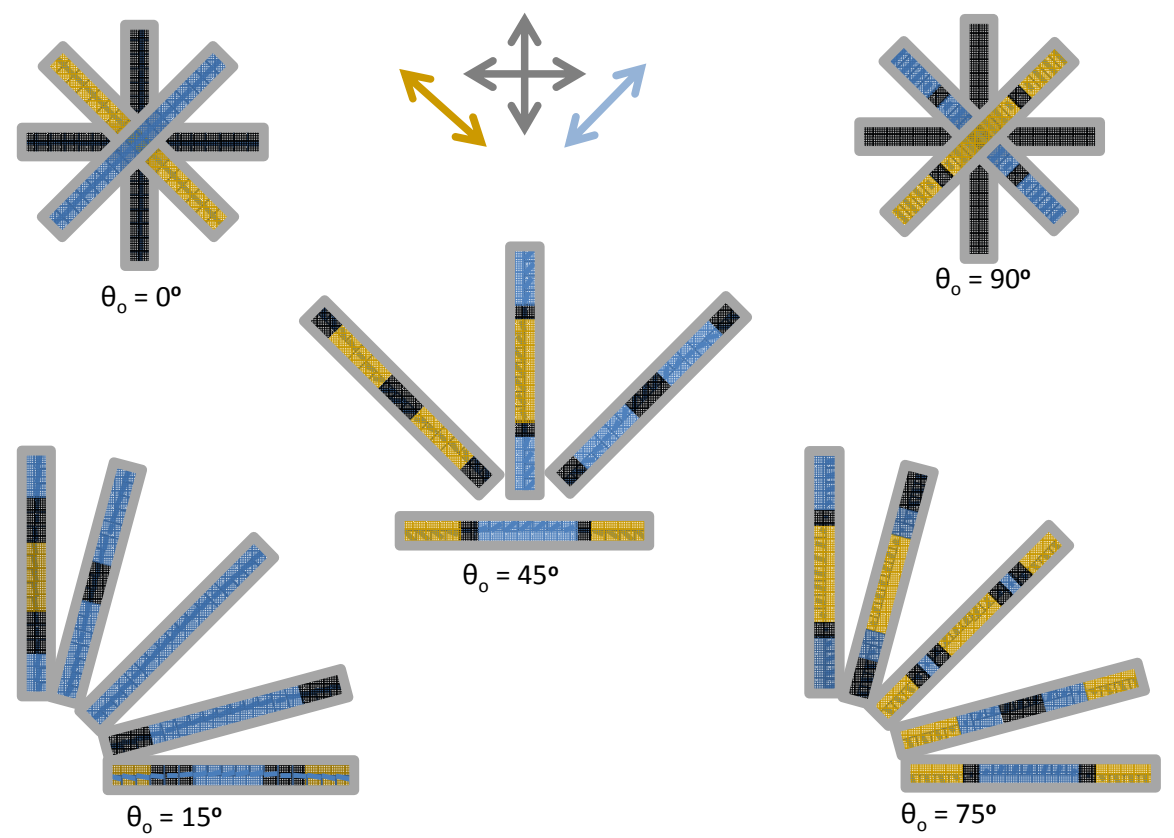

Figure S3. Colour patterns generated for a range of tilt angles from $\theta_{0}=$ $0^{\circ}$ (nematic) to $\theta_{0}=90^{\circ}$ (chiral nematic). The helix axes are assumed to be in the plane of the sample at the orientations shown. 


\section{Evidence for shear induced band formation for $9.6 \%$ aqueous suspensions of CNC}

When lyotropic or thermotropic liquid crystalline phases of stiff-chain polymers are subjected to shear, and then allowed to relax, bright and dark alternating lines perpendicular to the shear direction often appear. The reason for the shear-induced band structure is not entirely understood $^{2}$, but is clearly related to the viscoelasticity and liquid crystalline properties of the polymer solutions. The concentrated CNC suspensions also show liquid crystalline and viscoelastic properties ${ }^{3}$, so it seemed likely that they would also display shear-induced bands. Such bands were observed if the sample was viewed quickly after preparation. The bands were perpendicular to the direction of shear applied to the sample. (Figure S4).

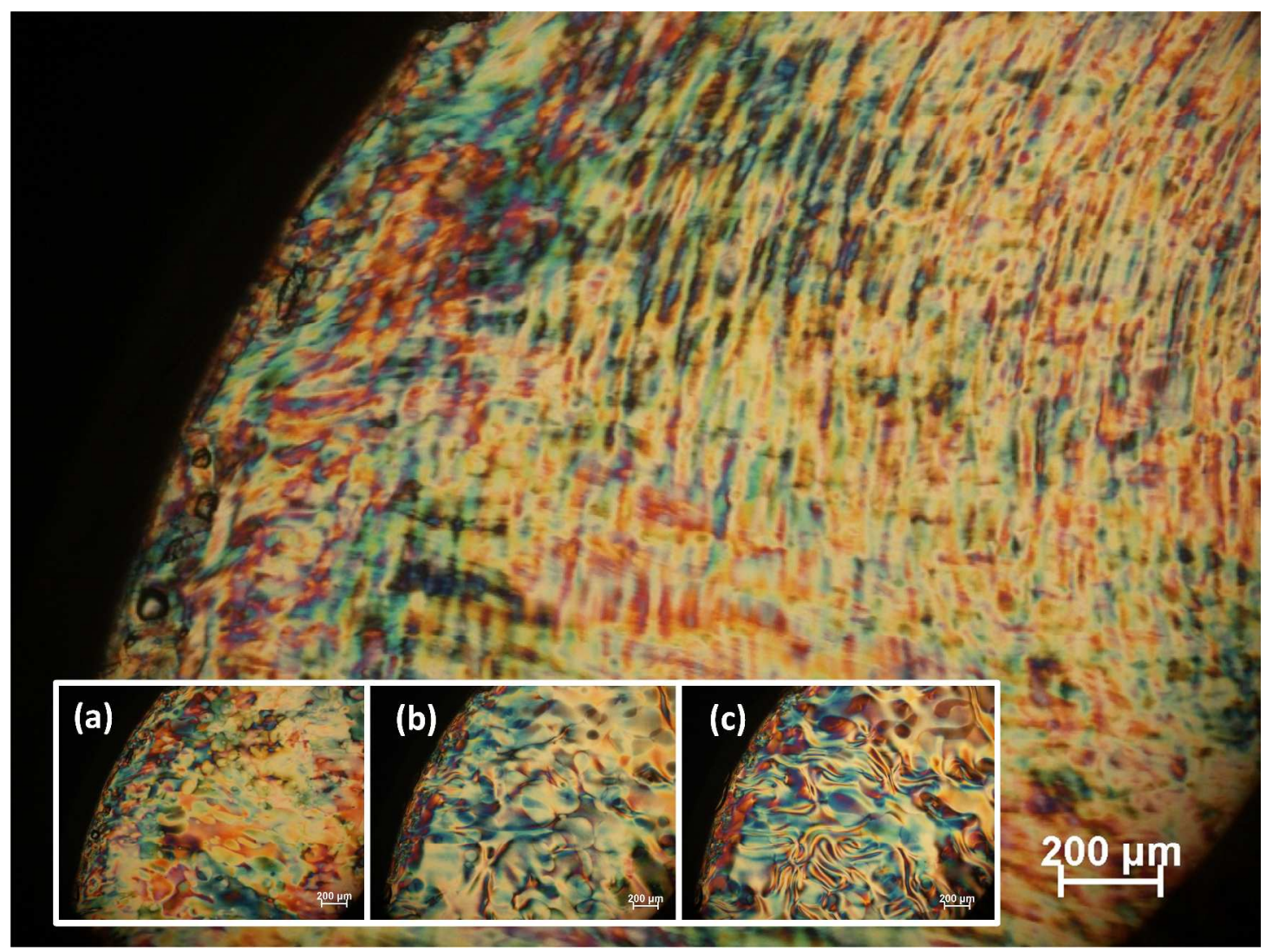

Figure S4. Shear-induced band structure for a droplet of CNC suspension (9.6 wt \%) sandwiched between microscope slide and cover glass. Pressure applied to the cover glass generated a radial flow of the suspension. The band direction is perpendicular to the flow, and the image was taken as soon as possible after sample preparation. The bands rapidly disappear; traces were still visible after $30 \mathrm{sec}$, but the usual transition from nematic to chiral nematic textures soon became apparent. The inserts show the same area after (a) 15 min., (b) 90 min., (c) 150 min. 


\section{REFERENCES}

(1) Borshch, V.; Kim, Y. K.; Xiang, J.; Gao, M.; Jákli, A.; Panov, V. P.; Vij, J. K.; Imrie, C. T.; Tamba, M. G.; Mehl, G. H.; Lavrentovich, O. D. Nematic twist-bend phase with nanoscale modulation of molecular orientation. Nat. Commun. 2013, 4, 2635.

(2) Kiss, G.; Porter, R.S. Flow induced phenomena of lyotropic polymer liquid crystals: the negative normal force effect and bands perpendicular to shear. In Mechanical and Thermophysical Properties of Polymer Liquid Crystals, Brostow, W. Ed.; Springer, New York, 2013, 380-404.

(3) 3 Ureña-Benavides, E. E.; Ao, G.; Davis, V. A.; Kitchens, C. L. Rheology and Phase Behavior of Lyotropic Cellulose Nanocrystal Suspensions. Macromolecules 2011, 44, 8990-8998. 